\title{
Impacto en salud infantil del invierno 2002: disociación entre factores ambientales y virus respiratorio sincicial, en Santiago
}

\author{
Luis F Avendaño $C^{1}$, Jorge Parra Va, Carolina Padilla Va, \\ María Angélica Palomino $M^{2}$. \\ The influence of respiratory virus \\ infections and environmental \\ conditions on pediatric health care \\ demand during winter-2002 \\ in Santiago, Chile
}

Background: The increase in winter related health pediatric demand is associated with three factors: cold and rainy weather, air pollution and respiratory viral epidemics. During the winter of 2002 there was, successively, heavy rain, air pollution, cold weather and a respiratory syncytial virus (RSV) epidemic in Santiago. Aim: To study the influence of environmental factors and RSV epidemic on pediatric health care demand. Patients and methods: The number of hospital admissions and outpatient consultations for acute lower respiratory infections (ALRI) from April to October 2002 (Autumn to Spring in Chile), were registered in a public pediatric hospital of Santiago. A respiratory virus surveillance (RSV, adenovirus influenza and parainfluenza virus) was carried out among children admitted for ALRI. Climate conditions and air pollution (number of particles of more than 10 microns/mm3) values were provided by local environmental health services. Results: As expected, a rise in winter hospital admissions and outpatient consultations was detected, that peaked in week 29. This rise coincided with the higher RSV detection week. There was heavy rain in weeks 22, 23 and 30 , as well as cold weather in June-July (weeks 23, 24, 26, 27 and 30; mid Winter), that did not increase health care demand. Likewise, high air pollution, registered from weeks 24 to 26 , did not increase health care demand. Conclusions: RSV epidemic is the principal factor associated to the increase in health burden during winter in Santiago (Rev Méd Chile 2003; 131: 902-8).

(Key Words: Health care costs; Environmental exposure; Respiratory Syncytial virus infections)

\footnotetext{
Recibido el 3 de marzo, 2003. Aceptado en versión corregida el 5 de junio, 2003.

Trabajo parcialmente financiado por Proyecto FONDECYT \# 1010630.

${ }^{1}$ Programa de Virología. ICBM. Facultad de Medicina. Universidad de Chile. ${ }^{2}$ Hospital Roberto del Río. Departamento de Pediatría. Facultad de Medicina, Universidad de Chile.

aEstudiante Carrera de Medicina, Universidad de Chile.
}

Correspondencia a: Luis F Avendaño, MD. Programa de

Virología. ICBM. Facultad de Medicina, Universidad de Chile. Av. Independencia 1027. Santiago. Chile. Fono: 5626786317.

Fax: 562678 6124. E mail: lavendan@machi.med.uchile.cl 
L as infecciones respiratorias agudas representan un problema prioritario de salud a nivel mundial, pues inciden fuertemente en la morbimortalidad infantil ${ }^{1,2}$. Su frecuencia aumenta todos los años durante los meses fríos, concitando un aumento de la atención de salud, tanto ambulatoria como hospitalaria ${ }^{3,4}$. Las infecciones respiratorias agudas bajas (IRAB), -que incluyen bronconeumonías, síndrome bronquial obstructivo, bronquiolitis, neumonitis, entre otras- tienen un rol protagónico como causa de hospitalizaciones y representan la primera causa de mortalidad infantil tardía. Diversos estudios han asociado las IRAB infantiles, y también las de adultos, con brotes de virus respiratorios, especialmente virus respiratorio sincicial (VRS) e influenza-9. Los avances en diagnóstico y caracterización viral han permitido definir el rol etiológico de estos virus en las diversas formas de presentación clínica $\mathrm{y}$ epidemiológica de las infecciones respiratorias ${ }^{10-16}$.

Durante las estaciones frías aumentan las lluvias y baja la temperatura ambiental en Santiago, lo cual induce cambios de costumbres en la población que pueden favorecer la propagación de infecciones respiratorias. Es posible que estos factores ambientales también incidan en la mayor sobrevida de agentes transmisibles en el ambiente, como se ha demostrado con el VRS, que en países tropicales aumenta en las temporadas lluviosas $^{17,18}$. Los cambios climáticos invernales, unidos a condiciones geográficas locales, favorecen el aumento de la contaminación en muchas ciudades del mundo, siendo Santiago un buen ejemplo de esta situación ${ }^{19-23}$.

La presencia simultánea en invierno, en Santiago, de los factores mencionados hace difícil evaluar independientemente el impacto en salud de cada agente 24,25 . Sin embargo, en 2002 la naturaleza parece haber decidido colaborar en dilucidar el problema presentando en forma sucesiva los factores lluvia, contaminación ambiental, frío y epidemia de VRS. Nuestra hipótesis es que las epidemias virales constituyen la principal causa de aumento de morbilidad estacional por afecciones respiratorias. Nuestro objetivo fue analizar el impacto de algunos factores ambientales y virales en la demanda de atención infantil abierta y cerrada en un área de salud de Santiago.

\section{Material y MÉTOdo}

Pacientes. El número de pacientes entre 0 y 15 años de edad hospitalizados por IRAB en el hospital de niños Roberto del Río, en la zona norte de salud de Santiago, Chile, entre abril y septiembre se obtuvo del Departamento de Estadística de dicho hospital. Se incluyeron los egresos con diagnóstico de neumonía, bronconeumonía, síndrome bronquial obstructivo, bronquiolitis, neumonitis y pleuroneumonía. El número de consultas al Servicio de Urgencia de este hospital, en pacientes de igual edad y con los mismos diagnósticos, se obtuvo de la misma fuente. Estas cifras se presentan por semana epidemiológica.

Estudio virológico. Desde 1998 a la fecha se está realizando una vigilancia en lactantes menores de 2 años, previamente sanos, que ingresan al hospital Roberto del Río por IRAB, enrolando entre 25 y 60 casos mensuales (aprobado por Comité de Etica de la Facultad de Medicina, Universidad de Chile). Se toma una muestra de aspirado nasofaríngeo antes de cumplir $48 \mathrm{~h}$ del ingreso y se procesa para inmunofluorescencia indirecta (IFI) para VRS, adenovirus, influenza y parainfluenza; se usan anticuerpos monoclonales proporcionados gentilmente por L Anderson (CDC, Atlanta USA) y P Pothier (Dijon, Francia) y conjugado comercial (SIGMA). Además se inoculan líneas celulares HEp-2 para aislamiento de VRS, adenovirus y parainfluenza y MCDK para virus influenza. Los cultivos se examinan por 7 días en busca de efecto citopático viral (ECP); a todos los cultivos se les hace IFI confirmatoria, haya o no ECP. Los virus detectados se tipifican posteriormente con anticuerpos monoclonales para definir VRS grupos A o B, influenzas A o B, parainfluenzas 1, 2 ó 3. En el presente estudio se presentan sólo los porcentajes semanales de detección de VRS, pues los otros virus no constituyeron brotes significativos durante 2002.

Contaminación aérea. Como indicador de contaminación aérea se considera la cifra diaria máxima de partículas $\geq 10$ micrones $/ \mathrm{m}^{3}$ (MP10) registrada en el Centro Censor del Servicio de Salud del Medio Ambiente (SESMA) de la Región Metropolitana. En este estudio se consideraron los valores de la Estación 
La Paz, ubicada en la zona norte de Santiago comespondiente al hospital Roberto del Río.

Temperatura diaria. La información sobre temperaturas diarias, por hora, de la Región Metropolitana se obtuvo de la oficina metropolitana de la Corporación Nacional del Medio Ambiente (CONAMA). Para los efectos de este estudio se calculó la temperatura promedio diaria usando el programa Excel.

Humedad diaria y lluvias. La información se recolectó de CONAMA RM. Se consideró el promedio diario de la humedad relativa del aire (\%) y en relación a lluvia se registraron los $\mathrm{mm}$ diarios de agua caída.

Estadística. Para el estudio de asociaciones entre indicadores de demanda de atención, variables climáticas y MP10 se determinaron coeficientes de correlación usando el programa estadístico MEDCALC 7.0. Se utilizó la probabilidad de 0,05 para definir la significación.

\section{RESULTADOS}

Durante el período de estudio, de abril a octubre de 2002 (semanas 14 a 44), se registraron 16.947 consultas por IRAB en el Servicio de Urgencia, con promedio de 546 semanales. Se observó un aumento de estas consultas, considerando una cifra arbitraria límite de 500 consultas semanales, entre las semanas 27 (junio, 30) y 40 (septiembre, 29), alcanzando el máximo en la semana 29 (julio, 14). En estas 31 semanas de estudio se registraron 531 hospitalizaciones por IRAB, con promedio semanal de 17. El número de hospitalizaciones por IRAB sobrepasó la cifra de 20 por semana entre las semanas 28 (julio, 7) y 36 (septiembre, 7) con un máximo observado en la semana 29 (Figura 1).

La vigilancia de virus respiratorios en el período abril-octubre se realizó en 256 lactantes menores de dos años ingresados por IRAB. Se detectaron los siguientes virus: 135 VRS (52,7\%), 2 adenovirus (0,8\%), 1 influenza $(0,4 \%)$ y 9 parainfluenzas (3,5\%). La epidemia de VRS duró 17

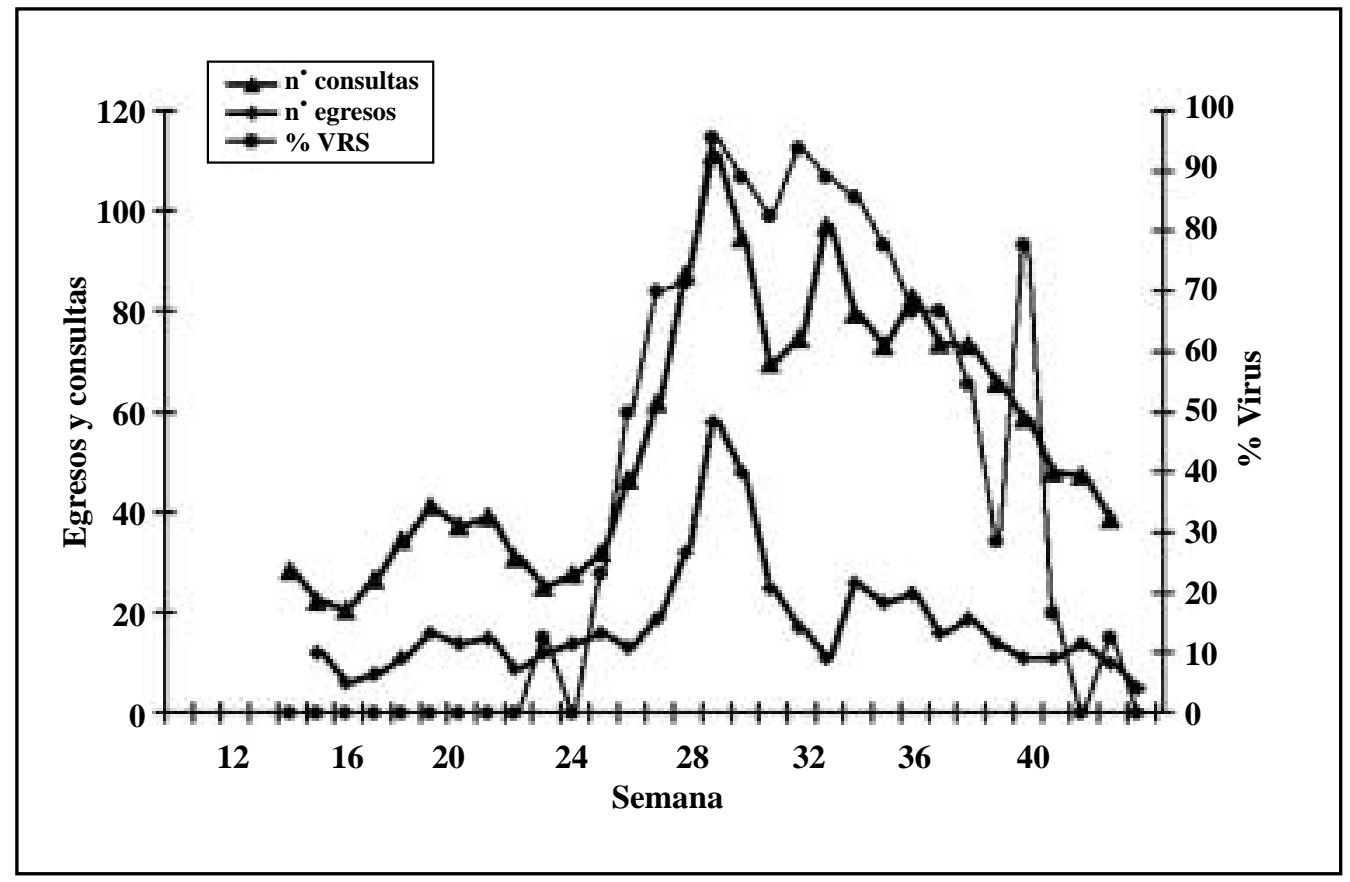

Figura 1. Relación entre el número semanal de egresos y consultas al Servicio de Urgencia (en decenas) por infección respiratoria aguda baja (IRAB) y el porcentaje de detección de VRS en lactantes hospitalizados por IRAB. Hospital Roberto del Río, Santiago, abril-octubre 2002. 


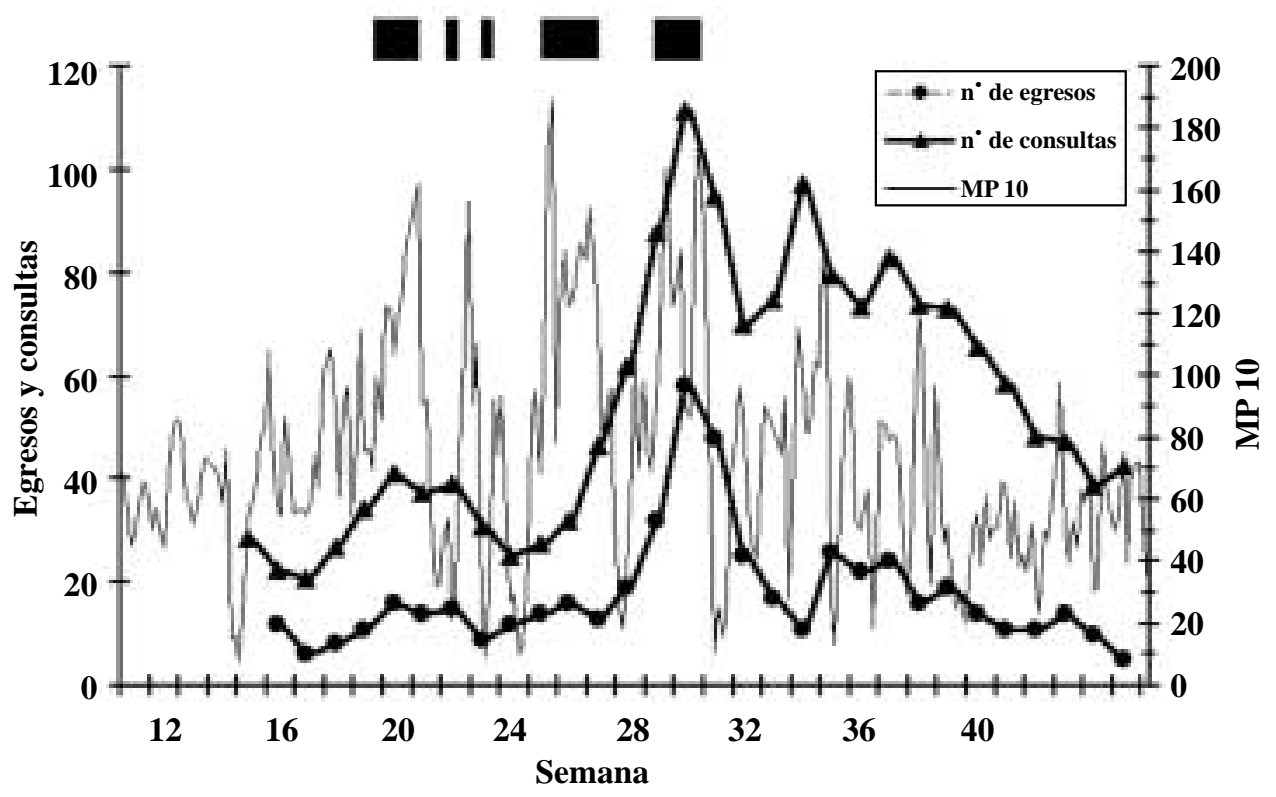

Figura 2. Relación entre el número semanal de egresos y consultas al Servicio de Urgencia (en decenas) por IRAB en el Hospital Roberto del Río y la contaminación aérea particulada (MP10) en el área norte de Santiago. Abril-octubre 2002. Los estados de alerta y pre-emergencia ambientales se ilustran mediante una barra en la parte superior de la figura.

semanas -considerando detección semanal sobre 20\%- y abarcó desde la semana 25 (junio, 16) hasta la 41 (octubre, 12), registrándose un valor máximo de 95\% en la semana 29. (Coeficiente de correlación r entre \% detección semanal de VRS y consultas por semana al Servicio de Urgencias: $\mathrm{r}=0,85 ; \mathrm{p}<0,0001$; IC $95 \%$ para $\mathrm{r}=0,72$ a 0,93 ) (Figura 1).

La contaminación ambiental, medida por el índice MP10 mostró una curva irregular, registrándose 38 días con niveles sobre 100, de los cuales 10 días tuvieron valores sobre 150. Las semanas 19 (mayo, 5), 25 (junio, 16) y 28 (julio, 7) fueron las más críticas, con 6 ó 7 días de MP sobre 100. En la Región Metropolitana se decretó alerta ambiental en 22 días y pre-emergencia ambiental en 11 días, por aumentos del índice ICAP sobre 100 y 300, respectivamente. Estos períodos críticos se concentraron en tres ciclos continuos: 4 al 12 de mayo (semana 19), 12 al 24 de junio (semanas 24 a 26), 9 al 18 de julio (semanas 28 y 29). En relación a la demanda de atención de salud, sólo la semana 19 se asoció a aumento no significativo de la consulta ambulatoria, no habiendo correlación entre ambos parámetros en el resto del período (coeficiente de correlación r entre número de días con PM10 $>100$ por semana y consultas al Servicio de Urgencias semanales: $r=0,0981 \mathrm{p}=0,6061$ (NS) IC $95 \%$ para $r=0,2718$ a 0,4427 ) (Figura 2 ).

Respecto de la temperatura ambiental, se observó una onda de temperatura media diaria más baja entre las semanas 22 (mayo, 26) y 31 (agosto, 3). Temperaturas semanales bajo $9,5^{\circ} \mathrm{C}$ se registraron en las semanas 23, 24, 26, 27 y 30 correspondientes a junio y julio. Esta onda fría coincidó parcialmente con el aumento de consultas por IRAB iniciado en la semana 27; sin embargo, las semanas 28 y 29 que tuvieron alta demanda en salud no registran temperaturas bajas. (Coeficiente de correlación $r$ entre temperatura media semanal y consultas al servicio de ungencias semanales: $r=0,0214 p=0,9173$ (NS) In- 
tervalo de confianza 95\% para $\mathrm{r}=-0,36$ a 0,40 ) (Figura 3).

El 2002 fue un año lluvioso, registrándose en Santiago total de $437 \mathrm{~mm}$ de agua caída, comparado con un año normal de $312 \mathrm{~mm}$. Los períodos de máxima lluvia fueron del 25 al 27 de mayo (semana $22=110 \mathrm{~mm}$ ), 2 al 5 de junio (semana $23=179 \mathrm{~mm}$ ) y 20 al 23 de julio (semana $30=74 \mathrm{~m}$ ). La humedad ambiental mostró valores fluctuantes y sólo se registró una onda de aumento sobre $80 \%$ en 4 de 7 días de la semana 22 (datos no mostrados), lo que correspondió a una baja demanda asistencial en salud. No hubo correlación entre porcentaje de humedad ambiental y presencia de lluvia (Figura 3).

\section{Discusión}

Las infecciones respiratorias son la principal causa del aumento de la patología respiratoria aguda, y en ellas la etiología viral juega un papel relevan$t^{2,6,14,18}$. En su patogenia intervienen factores de- pendientes del agente, del ambiente y del huésped. En este trabajo analizamos algunos aspectos de los dos primeros. En efecto, sólo consideramos el VRS por haber sido el virus más importante en el invierno de 2002, sin profundizar en aspectos de variación antigénica de las cepas prevalentes, que pudieran jugar un rol en la forma de presentación del brote ${ }^{10,16,17}$. De los factores ambientales hemos considerado la temperatura, la presencia de lluvias y la contaminación aérea, admitiendo que hay muchos otros que pueden ser importantes ${ }^{21}$. Por otro lado, de la variedad de indicadores para medir el potencial impacto en salud, seleccionamos el número de consultas a un servicio de urgencia y de egresos por infección respiratoria aguda baja, en un hospital pediátrico, porque hay buenos registros en el sistema público de salud y porque representan un indicador sensible de afecciones agudas de rápida variación. Además, la amplia diseminación de las virosis respiratorias permite extrapolar los hallazgos de la vigilancia en lactantes hospitalizados en el área norte de salud de Santiago, a toda la Región Metropolitana ${ }^{7,25}$. En relación a contaminación aé-

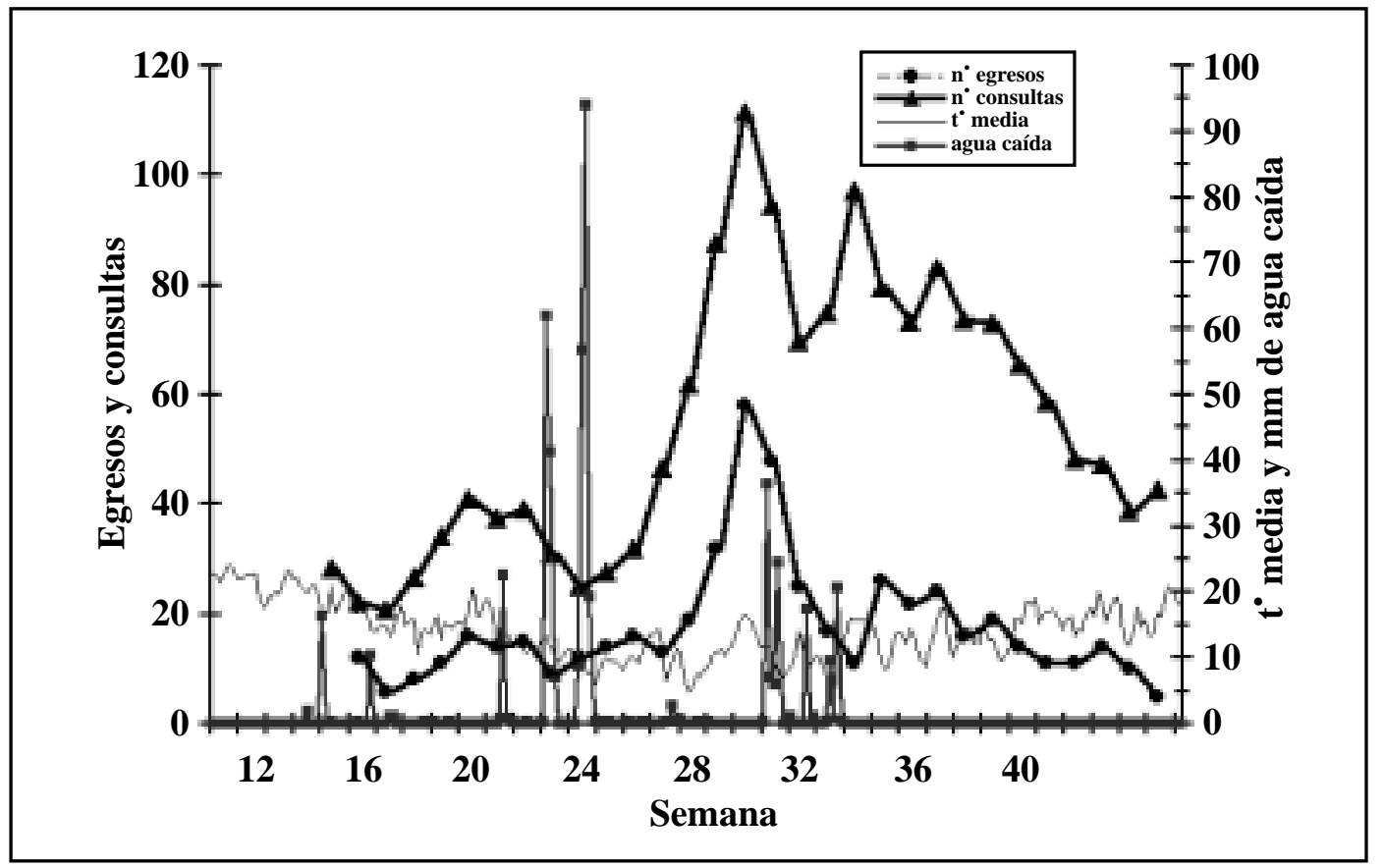

Figura 3. Relación entre el número semanal de egresos y consultas al Servicio de Urgencia (en decenas) por IRAB en el Hospital Roberto del Río, la temperatura media diaria y presencia de lluvia (mm agua caída diaria) en Santiago. Abril-octubre 2002. 
rea, se valoró sólo la información (MP10) consignada en una de las 8 plantas de medición del SESMA, la Estación La Paz, por corresponder al área geográfica en estudio y por considerarse generalmente representativa de la Región Metropolitana (E Gramsch. Comunicación personal. International Workshop. Health effects of air pollution, Santiago, 13-14 nov, 2002). Idealmente podrían considerarse MP2.5, gases y otros factores, que dejamos como futura tarea a los estudiosos de la contaminación. Como variables climáticas se seleccionaron temperatura media diaria y magnitud de lluvias ( $\mathrm{mm} /$ día), por su objetividad y facilidad de medición.

El presente trabajo confirma el impacto de las epidemias de virus respiratorios -en este año de VRSen la demanda de atención pediátrica, hecho observado periódicamente en la vigilancia montada desde $1988^{5,7,24}$. El virus que más incide sobre la salud infantil en estaciones frías es el VRS, pues mientras no se desarmolle una vacuna, su alta contagiosidad seguirá determinando infecciones en aproximadamente $60 \%$ de los niños que nace cada año, en todo el mundo ${ }^{17}$. La ausencia de un brote importante de influenza en 2002 realzó el papel que juega el VRS todos los inviernos en lugares de clima templado ${ }^{6-}$ 10,26. La duración y magnitud de los brotes invernales de VRS parecen depender del número de individuos susceptibles en la población y de las variaciones antigénicas del VRS, más que de las condiciones climáticas geográficas ${ }^{10,16,17}$. En efecto, las epidemias de VRS se presentan casi simultáneamente en la diversa geografía de Chile ${ }^{27-29}$. En este trabajo existe una comelación significativa entre detección de VRS y número de consultas y hospitalizaciones por IRAB. Incluso la doble onda detectada en la detección viral se refleja claramente en la demanda ambulatoria.

\section{REFERENCIAS}

1. Young P. White House to expand response to infectious diseases. ASM News 1996; 62: 450-1.

2. AvendaÑo LF. Resfrío común, influenza y otras infecciones respiratorias víricas. En: Meneghello J, ed. Pediatría. 5a ed. B Aires: Editorial Médica Panamericana, 1997; 1264-8.

3. Kaempfer AM, Medina E. Hospitalización infantil en Chile: Situación actual y perspectivas. Rev Chil Pediatr 1992; 63: 110-7.

4. KaEMPFER AM, Medina E. Análisis de la mortalidad
En 2002 la naturaleza contribuyó a dilucidar la importancia relativa de factores ambientales de los virales, pues primero azotó a Santiago con fuertes lluvias (179 mm en 4 días) que causaron inundaciones a principios de junio; luego hubo un aumento de la contaminación ambiental que obligó a las autoridades a decretar alarma y preemergencia ambientales entre el 12 y 24 del mismo mes; a continuación surgió una onda de frío a partir del 29 de junio. Sin embargo, recién en la semana 27 que se inicia el 30 de junio, empieza a aumentar el número de consultas y hospitalizaciones por IRAB, alcanzando el máximo en la semana 29, coincidiendo con la máxima presencia invernal de VRS. Posteriormente la demanda sigue una curva paralela a la de detección viral, sin influencia de los otros factores.

Creemos muy importante considerar el carácter multifactorial de la etiología de las enfermedades respiratorias, para tomar las medidas pertinentes y valorar sus efectos adecuadamente. Consciente de ello, el Ministerio de Salud ha implementado las «campañas de invierno», que han contribuido a disminuir la mortalidad infantil por neumonía ${ }^{25}$. En este contexto, creemos que el factor huésped, representado en este trabajo por niños, es el blanco más importante de las acciones preventivas, las que deben empezar por una clara definición clínica de la población en riesgo de enfermedad severa. Cada día hay más evidencias que a este grupo se están agregando los ancianos, los inmunocomprometidos y otros grupos ${ }^{8,9}$. La gran herramienta que representa la vacuna sólo tiene eficacia parcial en la prevención de influenza, pues en relación a VRS todavía queda camino por recorrer ${ }^{17}$.

infantil y factores condicionantes. Chile 1998. Rev Chil Pediatr 2000; 71: 405-12.

5. Avendaño LF, Larrañaga C, Palomino Ma, Gaggero A, Montaldo G, Suárez M et al. Community and hospital acquired respiratory syncytial virus infection in Chile. Pediatr Infect Dis J 1991; 10: 564-8.

6. Videla C, Carbalial G, Misiruan A, Aguilar M. Acute lower respiratory infections due to respiratory syncytial virus and adenovirus among hospitalized children from Argentina. Clinical and Diagnostic Virology 1998; 10: 17-23.

7. Lagos R, Avendaño LF, Levine M. Vigilancia 
sistemática de virus influenza, respiratorio sincicial, parainfluenza y adenovirus en niños ambulatorios con infecciones agudas respiratorias. Rev Méd Chile 1999; 127: 1063-72.

8. Dowell SF, Anderson LJ, Gary HE, ERdman DD, Plouffe JF, FILe TM et aL. Respiratory Syncytial Virus is an important cause of community acquired lower respiratory infection among hospitalized adults. J Infect Dis 1996; 174: 456-62.

9. FALSEY AR, WaLSH EE. Respiratory Syncytial Virus infection in adults. Clin Microbiol Rev 2000; 13: 371-.

10. Hall CB, Walsh EE, Schnabel KC, Long CE, McConnochie KM, HiLdreth SW et al. Occurrence of Groups A and B of Respiratory Syncytial Virus over 15 Years: Associated Epidemiologic and Clinical Characteristics in Hospitalized and Ambulatory Children. J Infect Dis 1990; 162: 1283-90.

11. Kajon A, Larrañaga C, Sú́rez M, Wadell G, Avendaño LF. Genome type analysis of Chilean adenovirus strains isolated in children's hospital between 1988 and 1990. J Med Virol 1994; 44: 16-21.

12. Kajon AE, Mistchenko AS, Videla C, Hortal M, WADELL G, Avendaño LF. Molecular epidemiology of adenovirus acute lower respiratory infections of children in the South Cone of South America (1991-1994). J Med Virol 1996; 48: 151-6.

13. ARENS M. Methods for subtyping and molecular comparison of human viral genomes. Clin Microbiol Rev 1999; 12: 612-26.

14. Larrañaga C, Kajon A, Vilagra E, Avendaño LF. Adenovirus surveillance on children hospitalized for acute lower respiratory infections in Chile (1988-1996). J Med Virol 2000; 60: 342-6.

15. Palomino MA, Larrañaga C, Avendaño LF. 2000. Hospital acquired adenovirus $7 \mathrm{~h}$ infantile respiratory infection in Chile. Pediatr Infect Dis J 2000; 19: 527-31.

16. Cane P. Molecular epidemiology of syncytial virus. Rev Med Virol 2001; 11: 103-16.

17. Cowns PL, McIntosh K, Chanock RM. Respiratory Syncytial Virus. En: Fields BN, Knipe DM, Howley PM, ed. Virology. Philadelphia. Lippincott-Raven Publishers, 1996; 1313-51.

18. Bedoya VI, Abad V, TrujLL H. Frequency of respiratory syncytial virus in hospitalized infants with lower acute respiratory tract illness in Colombia. Pediatr Infect Dis J 1996; 15: 1123-4.

19. Aranda C, Belmar R, Bello S, Muñoz M, Sheppard D, Oyarzún M. El problema de la contaminación atmosférica en la ciudad de Santiago. Enf Resp Cir Tor 1990; 6: 69-78.

20. Pope A, Dockery D. Acute health effects of PM10 pollution in symptomatic and asymptomatic children. Am Rev Resp Dis 1992; 145: 1123-8.

21. Seaton A, Mac Nee W, Donaddson K, Godden D. Particulate air pollution and health effects. Lancet 1995; 345: 176-8.

22. Rommieu I, Meneses F, Ruiz-Veiasco S, Sierra-Monje JJ, HuerTa J, White MC et al. Effects of air pollution on the respiratory health in Mexico City. Am J Resp Crit Care Med 1996; 154: 300-7.

23. Ostro BD, Eskeiand GS, Sánchez JM, Feyzioglu T. Air pollution and health effects: a study of medical visits among children in Santiago, Chile. Environ Health Perspect 1999; 107: 69-73.

24. Avendaño LF, Céspedes A, Stecher $X$, Palomino MA. Influencia de virus respiratorios, frío y contaminación aérea en la infección respiratoria aguda baja del lactante. Rev Méd Chile 1999; 127: 1075-9.

25. Girardi B, Astudiwo O, ZúÑIGa F. El programa IRA en Chile: hitos e historia. Rev Chil Pediatr 2001; 72: 292-300.

26. Vieira SE, Stewien K, Queiroz Dao, Durigon EL, TÖRÖKTH, ANDERSOn LJ ET AL. Clinical patterns and seasonal trends in respiratory syncytial virus hospitalization in Sao Paulo, Brazil. Rev Inst Med Trop S Paulo 2001; 43: 125-31.

27. Bancalari A, Martínez A, Casanueva P, Veliz F, Quinteros A, Walquer B et al. Etiología viral en la infección respiratoria aguda baja en recién nacidos. Rev Chil Pediatr 1999; 70: 201-7.

28. Alvarado M, Centeluas Z, Krstulovic J, Millar G, Pérez C, Poblete L et al. Experiencia local de salas IRA en la ciudad de Arica, años 2000-2001. Rev Chil Pediatr 2002; 73: 647.

29. Palomino ma, larrañaga C, Montaldo G, Suárez M, Díaz A, Avendaño LF. Infección extra e intrahospitalaria por virus sincicial respiratorio en lactantes. Rev Chil Pediatr 1994; 65: 11-6.

\section{Agradecimientos}

Agradecemos a Ignacio Olaeta (SESMA), Joyce Vera Bascour (CONAMA RM), Sonia Brintrup y Evelyn Llanquen (Departamento Estadística, Hospital Roberto del Río) por la colaboración en la recolección de la información respectiva. 